\title{
Multi Scale Aging Study of Copolymer PP/PE Matrix in Tropical Environment
}

Timothée Pertin ${ }^{1 *}$, Laurence Romana ${ }^{1}$, Anny Flory ${ }^{1}$

${ }^{1}$. Groupe de Technologie des Surfaces et des Interfaces - Université des Antilles - 97159 Pointe à Pitre - Guadeloupe - France - French West Indies

*Corresponding author: t.pertin@live.fr

Phenomenon happening at nanometric scale during aging of organic matrix composites are not fully understood mainly because of the spatial localization of the interphase region, which thickness ranges between 10 and $300 \mathrm{~nm}$ [1]. Development of investigation methods to study the evolution of nanometric properties of polymeric materials represents therefore a key point for these materials improvement regarding durability. Moreover, tropical climate is characterized by high temperatures, high relative humidity and solar irradiation up to $200 \mathrm{kWh} / \mathrm{m}^{2}$ [2]. Such conditions are extremely aggressive for polymeric materials in terms of aging and durability. International standards for lifetime prediction of most polymeric materials are also developed for continental or temperate regions and therefore, often conduct to underestimate the material lifetime in tropical environment. This misestimation contribute to the high plastic waste generation for the Caribbean region which was assessed to be around 1.5 million tons per year in 2010 [3]. Use of durable materials would contribute to reduce the amount of wastes and therefore generate savings for local actors.

In this study, chemical degradation and nano characterization protocols have been developed to investigate the correlation between structural changes induced by aging and the resulting mechanical properties of polymers at macro scale. The final aim of this work is to develop a fast characterization tools to predict polymer durability. Commercial thick PP/PE copolymer samples were aged using an artificial aging chamber up to 44 days of exposition. Chemical degradation was monitored by infrared spectrometry in Attenuated Total Reflection mode (ATR) and spectrums were analyzed. Scanning Electron Microcopy (SEM) was conducted to study surface topography of the samples. Nanomechanical properties were determined by continuous stiffness measurement (CSM) with indentation depths of $2 \mu \mathrm{m}$. Two setups were used to measure the nanomechanical properties. The indents were performed either on the exposed/unexposed faces or perpendicularly to the exposed face for the cross setup (Figure 1). Elastic modulus at macroscale was determined by Dynamical Mechanical Analysis (DMA).

Oxidation of the samples occurred after 3 days of aging and increased progressively up to 32 days. SEM observation showed apparition of surface cracks between 6 and 10 days of aging (Figure 2). More cracks that propagate through the sample were observed as aging time increases. Elastic modulus, $\mathrm{E}_{\mathrm{nano}}$, measured by nanoindentation on the exposed faces increased from $(1.35 \pm 0.04) \mathrm{GPa}$ at 0 days of aging to (3.6 \pm 0.2$) \mathrm{GPa}$ at 44 days (Figure $3 \mathrm{a}$ ). Cross setup measurements showed an exponential decrease of the Young's modulus with depth. The perturbation zone reached a maximal depth of $1300 \mu \mathrm{m}$ at 44 days. A Voigt model was used to calculate the Young's modulus of the bulk sample using nanoindentation data. This calculated parameter, $\mathrm{E}_{\text {macro fit, }}$ was then compared to the macro Young's modulus, $\mathrm{E}_{\text {macro, }}$ deduced from DMA tests (Figure $3 \mathrm{~b}$ ). A good correlation was observed at macro and nano scales as long as macroscopic defects such as cracks appeared. This work shows that nanoindentation is a suitable technique to detect early stage degradation of polymers and therefore a powerful tool to predict durability of polymeric materials. 
References:

[1] L. Riaño, L. Belec, J.F. Chailan, Composite Structures 198 (2018), p. 109.

[2] A. Bertin, J.P. Frangi, Energy Conversion and Management 75 (2013), p. 593.

[3] J.R. Jambeck, R. Geyer, C. Wilcox, Science 347 (2015), p. 768.

(a)

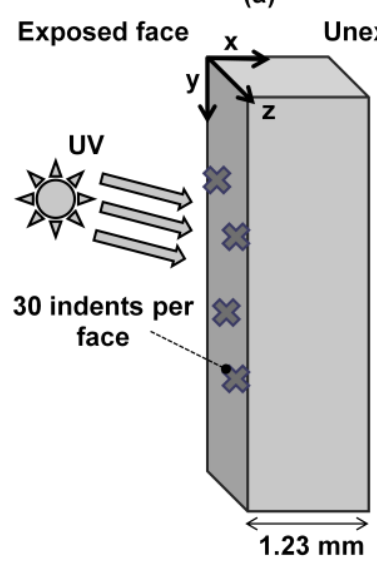

(b)

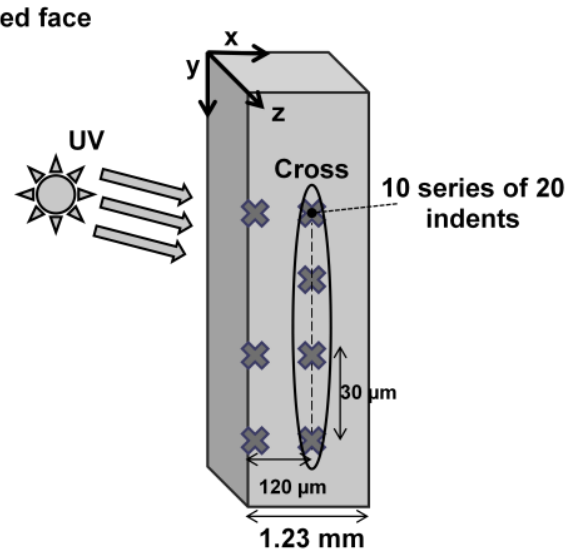

Figure 1. Indents localization for data acquisition for (a) normal setup and (b) cross setup.

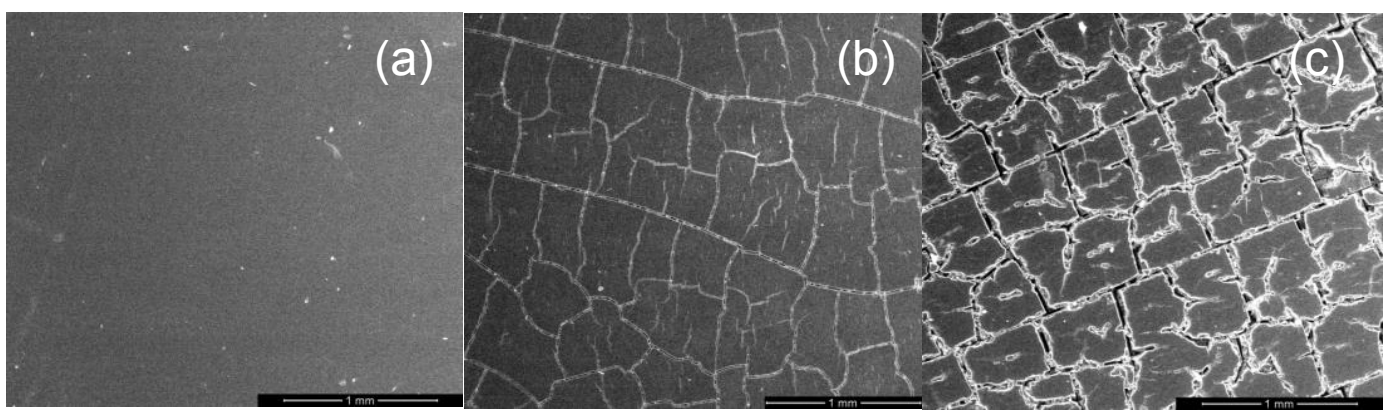

Figure 2. SEM photography of exposed face in SE mode at (a) 6 days of aging, (b) 18 days of aging and (c) 32 days of aging. Scale bar is $1 \mathrm{~mm}$, x100 magnification.

(a)

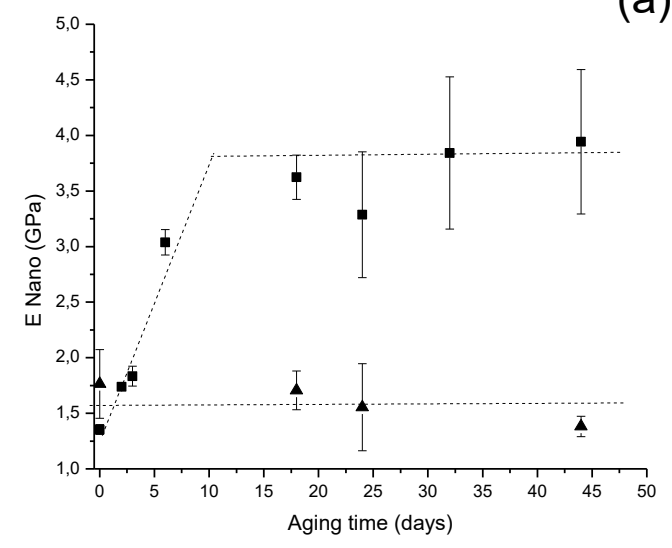

(b)

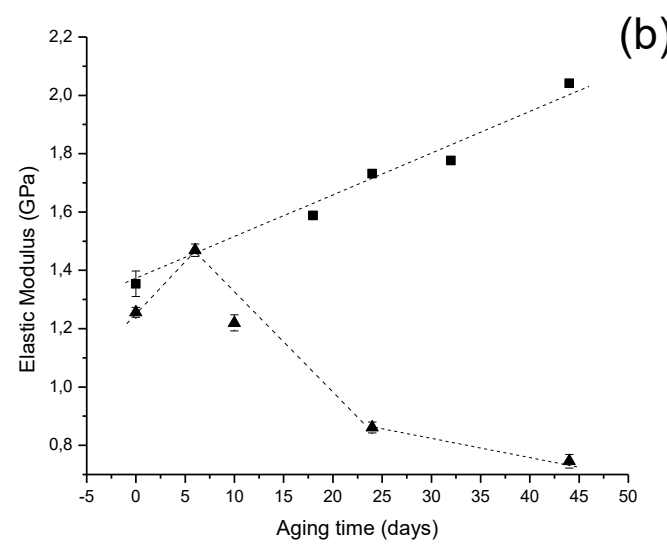

Figure 3. (a) Evolution of $E_{\text {nano }}$ measured on exposed (square) and unexposed (triangle) faces with the aging time. (b) Comparison of the evolution of $E_{\text {macro fit }}$ (square) and $E_{\text {macro }}$ (triangle) with the aging time. 